\title{
What is the Future of Intrauterine Surgery?
}

\author{
1,2,3 Renato A Moreira de Sá, ${ }^{3,4}$ Fernanda $C$ da Silva
}

Keywords: Fetal therapy, Fetal ultrasonography, Fetoscopic surgery, Minimally invasive surgery.

How to cite this article: Moreira de Sá RA, da Silva FC. What is the Future of Intrauterine Surgery? J Ultrasound Obstet Gynecol 2018;12(4):252-254

Source of support: Nil

Conflict of interest: None

\section{INTRODUCTION}

When reviewing the history of fetal surgery from its early years, its remarkable achievements strengthen its role in fetal medicine. In the 1960s, the human fetus was practically unknown and poorly studied. ${ }^{1}$ Ultrasound technology, from the 1970s onward, made possible most of our current understanding of fetal pathophysiology through imaging. Hence, during the past few decades ultrasound has generated a revolution in obstetrics. The last 40 years witnessed striking progress in fetal therapy as a result of the before coming advancements in imaging. ${ }^{2,3}$

While the standard treatment for most fetal anomalies still remains planned delivery at a tertiary center with appropriate neonatal therapy, currently, a number of fetal malformations can be treated with fetal surgery. A growing subset of anatomical anomalies may benefit from surgical intervention in the unborn fetus due to the advances in imaging modalities, instrumentation, and surgical techniques.

The first fetal surgeries took place in the 1980s and it was open surgery (maternal laparotomy, followed by hysterotomy with direct exposure of the fetus). However, as laparoscopic and video-assisted procedures became more available, open surgery is gradually being replaced by fetoscopy - a less invasive surgical technique. Fetoscopic surgery has progressed from a mere concept to an accepted and viable treatment option. ${ }^{4,5}$ The main reason for the growth of fetoscopy is that it avoids maternal

\footnotetext{
${ }^{1-4}$ Professor

${ }^{1}$ Department of Obstetrics, Federal Fluminense University, Brazil

${ }^{2}$ Fernandes Figueira Institute/Fundação Oswaldo Cruz, Brazil

${ }^{3}$ Perinatal Group, Brazil

${ }^{4}$ Rio de Janeiro State Federal University, Brazil
}

Corresponding Author: Renato A Moreira de Sá, Professor Department of Obstetrics, Federal Fluminense University, Brazil, e-mail renatosa.uff@gmail.com morbidity associated with a large laparotomy incision and possibly reduces the incidence of perinatal complications through the prevention of a large hysterotomy. Although strongly criticized during its early years, in the recent history of fetal medicine, a few pioneers foresaw the benefits of minimizing surgical trauma and the associated advantages to the patient, bringing fetoscopy to our attention. ${ }^{4-6}$

Despite the minimally invasive nature of fetoscopy, it is associated with iatrogenic preterm rupture of membranes and preterm labor. ${ }^{2}$ To move toward the future, we must overcome these challenges. Incorporating new technologies may help us on this journey. Some prospective solutions, based on technologies that are already under development, look promising. Research has revolved around preventing such complications as amniotic fluid leakage, preterm premature rupture of membranes and preterm labor. ${ }^{4-8}$ Iatrogenic preterm premature rupture of the membranes (PPROM) is a significant factor contributing to perinatal outcome. Even a small diameter puncture, due to disruption of the fetal membranes increases the risk fo PPROM. It has been proposed that the amniotic fluid leakage is related to the separation of the membranedecidua attachment with independent movement of the chorion and amnion. Considering this, we may suppose that the restoration of the integrity of membrane will require an exogenous matrix to occlude an iatrogenic defect in the membranes, creating a seal. Initial reports about an absorbable collagen sponge plug and, more recently, absorbable gelatin sponge as a membrane sealant following a fetoscopic procedure may provide an effective and safe means of reducing iatrogenic PPROM and thus, prolonging pregnancy following intervention. ${ }^{9,10}$ Nevertheless, the results so far have not proven that a lower rate of iatrogenic PPROM occurred after the use of either sealant method. Their use could be associated with a reduced procedure-to-delivery interval. Approaches to the closure of fetal membrane defects with liquid sealants or solid biomaterial scaffolds, self-adhesive ultrathin polymeric film, and new glues inspired by mussels focusing on prophylactic plugging of fetoscopic access sites remain under investigation. Further understanding of scarless healing may prevent not only fluid leakage but also early labor in fetal-surgery patients. ${ }^{10}$

Multiple potential therapies have been advocated for the treatment of PPROM with some success, especially in cases of post-procedural fetal membrane rupture. 
Promising research seeking to prevent bacterial infection after PPROM, avoiding preterm delivery, pulmonary hypoplasia and sepsis is the use of subcutaneously implanted intrauterine port systems that enable longterm, continuous lavage of amniotic cavity using hypotonic aqueous composition similar to human amniotic fluid combined with intra-amniotic application of antibiotic.

Although the use of basic laparoscopic techniques is the basis of minimally invasive fetal interventions, it is important to look for ways to avoid the limitations associated with fetoscopic procedures, such as a lack of tactile feedback, two-dimensional vision, and the limited degrees of freedom of the laparoscopic instruments. Robotic surgery has been available since łate 1990s, and today they have real potential to contribute to the development of fetal surgery. ${ }^{10}$ Robotic platforms are directed a surgeon situated a distance from the surgical field, using a three-dimensional laparoscopic view and special "chopstick" instruments. The hardware filters the motions of surgeon's hand and it is thought to provide increased precision and enhanced dexterity, allowing for multiple degrees of freedom, similar to the human wrist. ${ }^{10}$

With newer imaging modalities and developing technologies, the need for open surgery has decreased. Advances in videoendoscopic technologies and instruments may complement the use of image-guided in utero procedures. Further developments of higher-dimensional transducers and image software may expand the use of ultrasound in invasive obstetric interventions. ${ }^{11}$ In the 1970s, the development of imaging techniques leapfrogged the development of fetal medicine; in the same way, the development of multifunctional radiology and augmented reality may lead us into the future once again. Radiology may be the field in which we can expect to see the biggest progress in development as it is one of the areas in medicine which is fastest growing and developing. Using multifunctional radiology, a machine may be able to detect many medical problems and biomarkers. Such use could be an important progression in both imaging technology and interventional radiology. There is a new iPad app from Fraunhofer Institute for Medical Image Computing MEVIS in Germany which uses augmented reality technology to help surgeons. While operating surgeons can see through anatomical structures like blood vessels in the liver without opening organs and therefore they can perform more precise excisions. $^{12}$

In silico clinical trials can also contribute to the development of fetal therapy. Switching from clinical trials in obstetrics and in fetal medicine, which are extremely difficult due to to ethical issues, to clinical trials on tiny microchips used as models of human cells, organs, or whole physiological systems provides clear advantages. Using in silicoclinical trials, drugs or components could be tested without limitations and that, would make clinical trials faster and even more accurate; (in each case, the conditions and circumstances would be the same. The organs-on-chips technology can use stem cells to mimic organs of the body with a series of devices. There are many experts who believe that this technology could revolutionize clinical trials and replace animal testing completely. ${ }^{13}$

Treating disease in utero, and thereby facilitating the birth of a healthy infant, has multiple biological advantages unique to fetal development, which provides a compelling rationale for performing potentially curative treatments, such as stem cell transplantation or gene therapy, prior to birth. To date, in utero blood transfusions containing blood stem cells extracted from the mother's bone marrow is possible and is being used in fetal treatment. ${ }^{14}$

In the near future, there may also be ways to combine surgical techniques with stem cell therapy to treat defects even earlier, which might improve a child's outcome. If such a combination approach succeeds, this could be beneficial for children with spina bifida, for example. By patching the defect earlier the severity of side effects may be reduced. ${ }^{15}$

A significant number of congenital diseases can benefit from fetal surgery. As early as the 4th century, Hippocrates stated: "What medicines do not heal, the lance will...." ${ }^{16}$ While the history of fetal surgery demonstrates a magnificent evolution from past decades to the present, safety of a patient and medical ethics must be the cornerstone of the future of fetal surgery.

\section{REFERENCES}

1. Maselli KM, Badillo A. Advances in fetal surgery. Annals of translational medicine. 2016 Oct;4(20):394.

2. Kumar S, More M, Sharma SF, et al. Surgery: A Basic Overwiew and a Glimpse into Its Future. MGM Journal of Medical Sciences. 2017;4(1):35-38.

3. Graves CE, Harrison MR, Padilla BE. Minimally Invasive Fetal Surgery. Clin Perinatol. 2017 Dec;44(4):729-751.

4. Pedreira DAL. Advances in fetal surgery. einstein. 2016;14(1):110-112.

5. Pedreira DA, Zanon N, de Sá RA, et al. Fetoscopic singlelayer repair of open spina bifida using a cellulose patch: preliminary clinical experience. J Matern Fetal Neonatal Med. 2014;27(16):1613-1619.

6. Kohl T. Percutaneous minimally invasive fetoscopic surgery for spina bifida aperta. Part I: surgical technique and perioperative outcome. Ultrasound Obstet Gynecol. 2014;44(5):515-524.

7. Adzick NS, Thom EA, Spong CY, Brock JW 3rd, Burrows PK, Johnson MP, Howell LJ, Farrell JA, Dabrowiak ME, Sutton LN, Gupta N, Tulipan NB, D'Alton ME, Farmer DL; MOMS Investigators. A randomized trial of prenatal versus 
postnatal repair of myelomeningocele. N Engl J Med. 2011; 364(11):993-1004.

8. Pensabene V, Patel PP, Williams P, et al. Repairing fetal membranes with a self-adhesive ultrathin polymeric film: evaluation in mid-gestational rabbit model. Annals of biomedical engineering. 2015 Aug 1;43(8):1978-1988.

9. Tchirikov M1, Zhumadilov Z, Winarno AS, et al. Treatment of Preterm Premature Rupture of Membranes with Oligo-/ Anhydramnion Colonized by Multiresistant Bacteria with Continuous Amnioinfusion and Antibiotic Administrations through a Subcutaneously Implanted Intrauterine Port System: A Case Report. Fetal Diagn Ther. 2017;42(1):71-76.

10. Devaud YR, Züger S, Zimmermann R, et al. Minimally Invasive Surgical Device for Precise Application of Bioadhesives to Prevent iPPROM. Fetal Diagn Ther. 2018 Jun 19:1-9.

11. Nawapun K, Phithakwatchara N, Jaingam S, et al. Advanced ultrasound for prenatal interventions. Ultrasonography. 2018 Jul;37(3):200-210.
12. The Medical Futurist. 20 Medical Technology Advances: Medicine in the Future-Part II July, 14th, 2016. Available from https:/ / medicalfuturist.com/20-potential-technologicaladvances-in-the-future-of-medicine-part-i

13. The Medical Futurist. 20 Medical Technology Advances: Medicine in the Future-Part II July, 20th, 2016. Available from https:/ / medicalfuturist.com/20-potential-technologicaladvances-in-the-future-of-medicine-part-ii/

14. Götherström C, Hermerén G, Johansson M, et al. Stem cells and fetal therapy: is it a reality? Obstetrics, Gynaecology \& Reproductive Medicine. 2017 May 1;27(5):166-167.

15. Almeida-Porada G, Atala A, Porada CD. In utero stem cell transplantation and gene therapy: rationale, history, and recent advances toward clinical application. Molecular Therapy-Methods \& Clinical Development. 2016 Jan 1;3:16020.

16. Antoniou SA, Antoniou GA, Antoniou AI, et al. Past, present, and future of minimally invasive abdominal surgery. JSLS: Journal of the Society of Laparoendoscopic Surgeons. 2015 Jul;19(3). 